\title{
Erratum: Erbitux diagnostic latest adjunct to cancer therapy
}

Peter Mitchell

Nat. Biotechnol. 22, 363-364 (2004)

On page 363, column 2, line 14, sepsis is incorrectly listed as one of the side effects of Erbitux. The sentence should have read "potential side effects such as rare cases of interstitial lung disease." 'self-cleaving' 2A peptide-based retroviral vector

Andrea L Szymczak, Creg J Workman, Yao Wang, Kate M Vignali, Smaroula Dilioglou, Elio F Vanin \& Dario AA Vignali Nat. Biotechnol. 22, 589-594 (2004)

In Figure 3b, the labels for CD3e and CD3e-2A were inverted. Thus, the second band should have been labeled CD3e-2A and the third band labeled CD3e.

\section{Corrigendum: The potential environmental impact of engineered} nanomaterials

\section{Vicki L Colvin}

Nat. Biotechnol. 21, 1166-1170 (2003)

In the Acknowlegments, line 3, the name Cafer Yavuz was misspelled as Cafer Tevuyz.

\section{Addendum: Correction of multi-gene deficiency in vivo using a single 'self-cleaving' 2A peptide-based retroviral vector}

\section{Andrea L Szymczak, Creg J Workman, Yao Wang, Kate M Vignali, Smaroula Dilioglou, Elio F Vanin \& Dario AA Vignali} Nat. Biotechnol. 22, 589-594 (2004)

Technical note: Recent experiments with other 2A-linked constructs have suggested that cleavage efficiency can be influenced by the protein $\mathrm{NH}_{2}$-terminal to the $2 \mathrm{~A}$ peptide. In some instances, we have found that cleavage efficiency can be improved by placing a Gly-Ser-Gly linker between $\mathrm{NH}_{2}$-terminal protein and the $2 \mathrm{~A}$ peptide. 